\title{
The Design of Monitoring and Warning System for the Environment of Tropical Crop Growth based on Multi-sensor
}

\author{
Lingling Wang ${ }^{1,2, a}$ Yuping $\mathrm{Li}^{\star 1,2, b}$ Hongxia Luo ${ }^{1,2, \mathrm{c}}$ Jihua Fang $^{1,2, \mathrm{~d}}$ \\ ${ }^{1}$ Institute of Information and Technology, Chinese Academy of Tropical Agricultural Sciences, \\ Danzhou, 571737, China; \\ ${ }^{2}$ Key Laboratory of practical research on tropical crops information technology in Hainan, Danzhou, \\ 571737, China \\ aemail:zishi-010@163.com, bemail:xxsrdny@163.com \\ cemail:120081008@163.com, demail:fangdil@163.com, *corresponding author
}

Keywords: data monitoring; field environment; multi-sensor; tropical agriculture

\begin{abstract}
Agricultural information technology is an emerging research direction with high effect agriculture developing. The paper proposes a design idea on monitoring and warning system for the environment of tropical crop growth based on technology of multi-sensor, database, computer interface, embedded programming, wireless sensor network, etc. The paper studies data criterion, tropical crop environment monitoring system, monitoring and early warning platform, and information system integration platform. The research mainly explores the relative importance of tropical crop growth and field environment, which have great significance in conducting the cultivation for high yielding, good quality and high efficiency.
\end{abstract}

\section{Introduction}

Tropical crop is an important part of agriculture in China. According to the national conditions, China's tropical agricultural development has entered into a new era which transforms the traditional agriculture into high-output, good-quality and high-efficiency agriculture at this stage, but how to use advanced information technology to remodel traditional is a great subject.

A few studies on tropical crop growth environment have been done in our previous work ${ }^{[1-3]}$, but the data is limited and inconclusive. We find that environmental condition is complex greatly affected the growth of crop in the tropics through exploration. Monitoring and warning on field meteorological disasters is lack during tropical crop growth such as drought, flood and typhoons. In the research, monitoring and warning system for the environment of tropical crop growth based on multi-sensor is designed to help solving the problem of important data acquiring, data accuracy, data transfer and data use.

\section{Data Criterion Research}

The paper studies field environment data standard on tropical crop to analyze the description method of farm environmental factors, based on database standard, agriculture informatization standard and farm environment standard. The data standard includes national standard, industry standard and local standard. The data consists of two sections, field environment data and crop nutrient data, including wind velocity and direction, light intensity, air humidity, air temperatures, soil moisture, the width of leaf, height, breadth, diameter of stem, length of internode, number of leaves, etc. The final aim is to establish a standard database management system, which can unified managing and investigating the field investigation data and ownership, analyze the data flow through each process, and present the data in standard way to different users. The research idea of data criterion is as shown in Figure 1. 


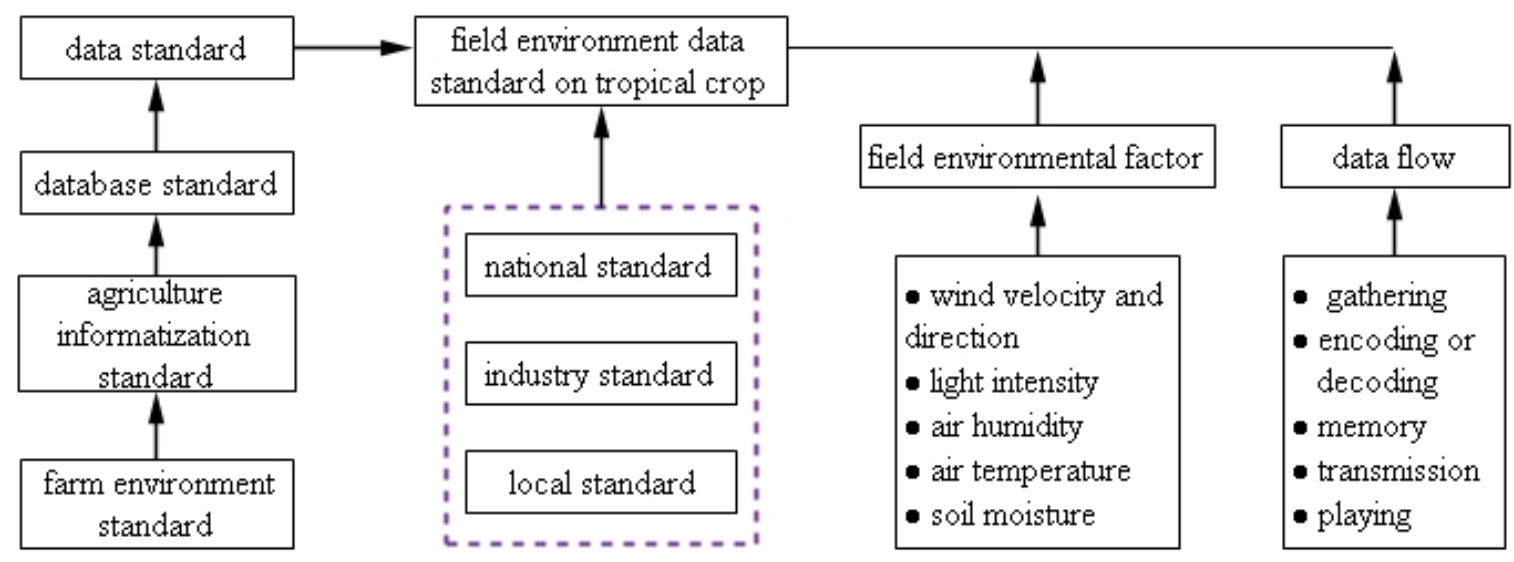

Fig.1. The research idea of data criterion

\section{Tropical Crop Environment Monitoring System Design}

Based on the technology of multisensor fusion, computer interface, embedded programming, database, wireless sensor network and mathematical model ${ }^{[4-6]}$, a design of tropical crop environment monitoring system is given. The main research emphasis of image special sensor, soil special sensor, meteorological special sensor, plant growth sensor and hybrid sensor, which can be suitable for both tropical crop in greenhouse and in open field. Tropical crop environment monitoring system structure is as shown in Figure 2. The system design is as shown in Figure3.
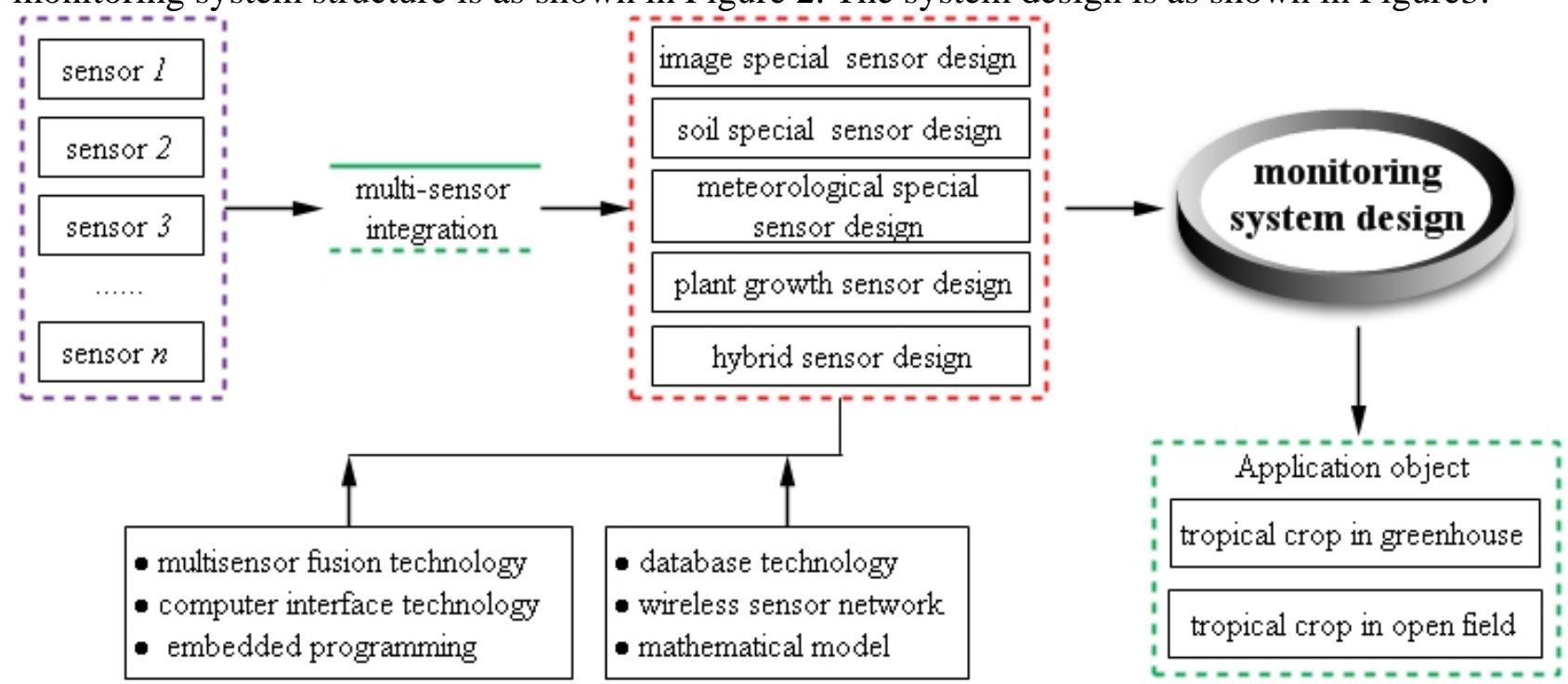

Fig.2. Tropical crop environment monitoring system structure
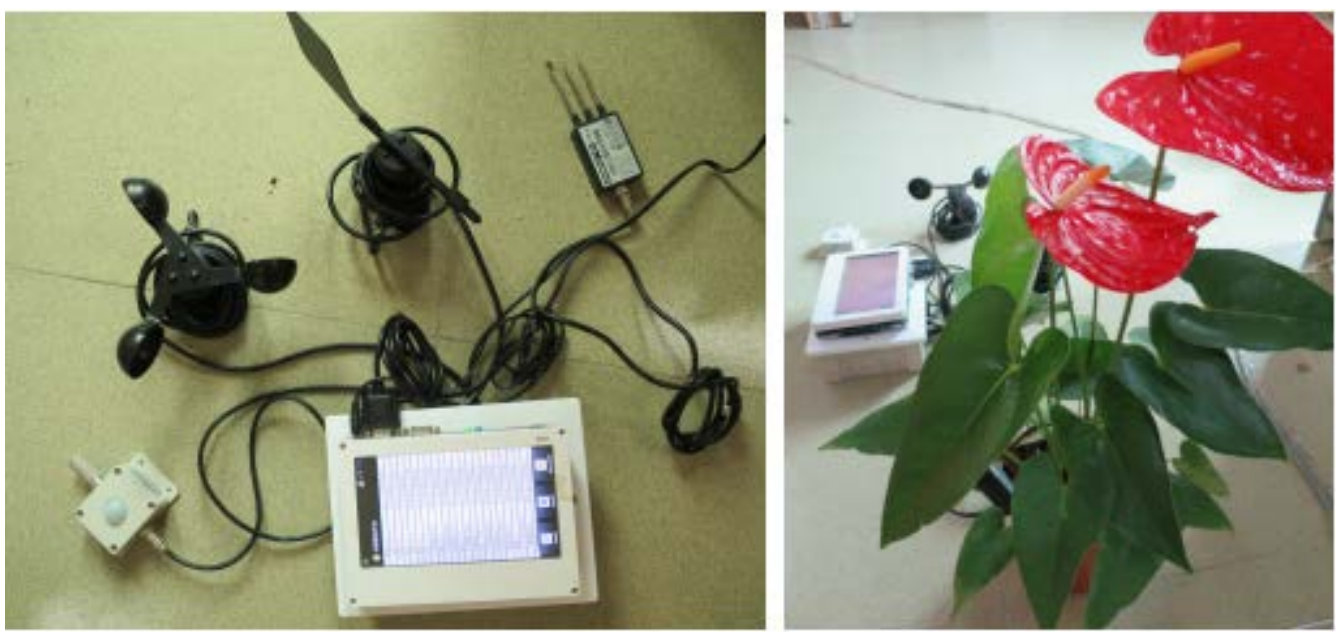

Fig.3. Tropical crop environment monitoring system design 


\section{Monitoring and Early Warning Platform Design}

Database of monitoring tropical crop growth environmental quality of farm field is the new style of managing farm field data and monitoring environment quality. With database and wireless sensor network technology, monitoring and early warning platform can be built. The monitoring data includes crop image, meteorological and environment, crop growth and nutrition, etc. The monitoring method includes remote monitoring, field monitoring and laboratory monitoring. The human-machine interaction module can show the data in various ways, including data curve, table, thematic map and animate simulation, and has the same parameters setting style and the safe control platform. The exact space expression is also explored. Based on the database, the show of statistical analysis, anomaly recognition and trend analysis can help to make decision on the suitability evaluation of tropical crop growth environment. Monitoring and early warning platform structure is as shown in Figure 4.

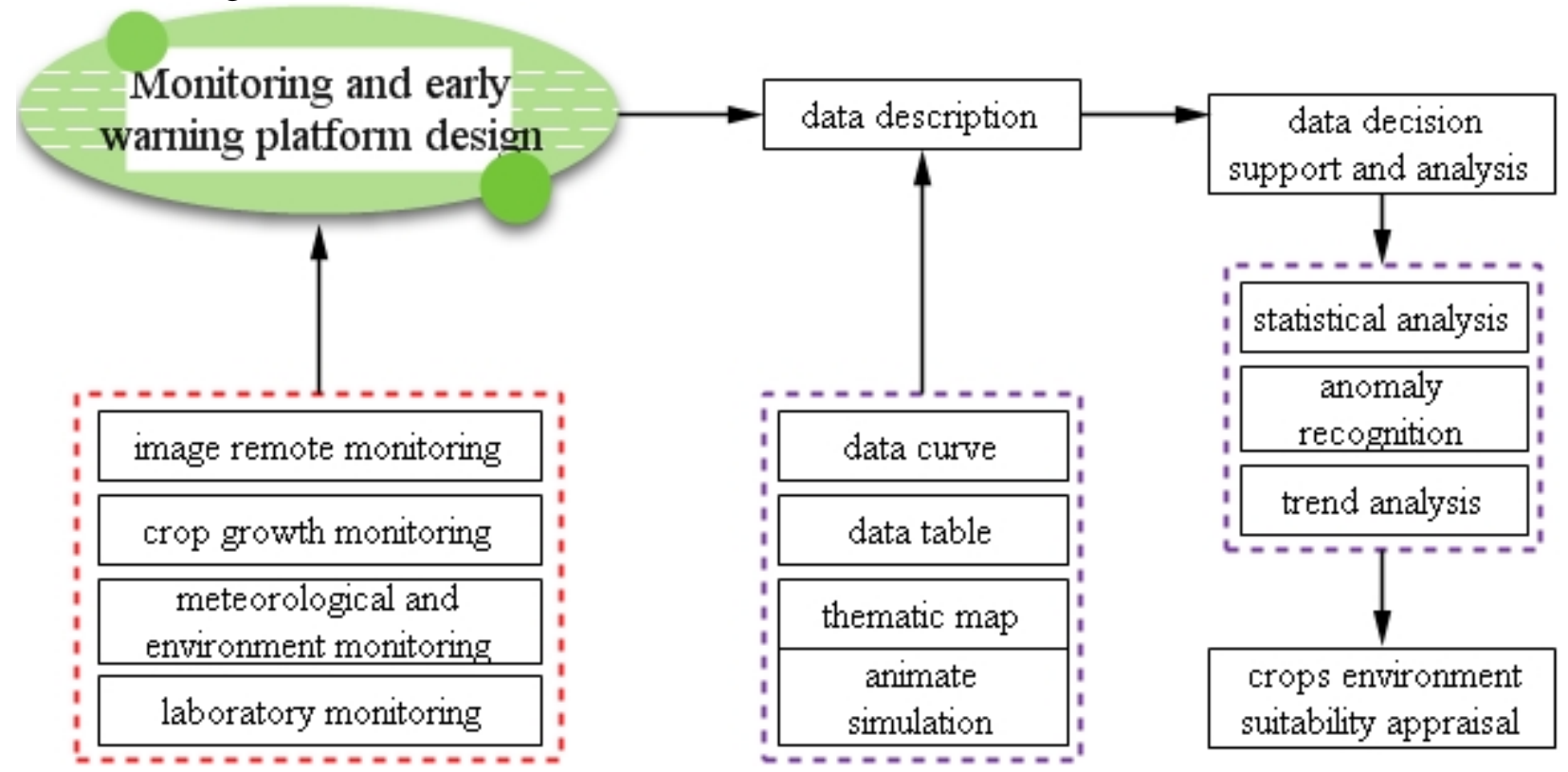

Fig.4. Monitoring and early warning platform structure

\section{Information System Integration Platform Design}

The seamless joint of tropical crop environment data acquisition equipment and database is crucial for information system integration platform design. This platform can stably achieve the dependable access and rapid acquirement of data using advanced instruments such as automatic meteorological station, portable soil monitoring instrument, integrated sensor, webcam etc. Through summarization and organization of function principle solutions and contradiction resolving principles on tropical crop growth environment information, database is established hierarchy, including environment database, nutrient database, image database and history database. Data collection in many ways improves the accuracy and reliability of the database. Analysis and application be achieved in two ways by information system integration platform. One is online analytical process and early warning, and the other is typhoon, drought and floods disaster preparedness and response. Information system integration platform design is as shown in Figure 5.

\section{Conclusion}

The research on monitoring and warning system for the environment of tropical crop growth has tropical distinctive regional feature, which melts into new ideas, new methods and new technology. The platform design is an important feature for exploring the order and the patterns between tropical crop growth and field environment. The work supports the theory and technological base for crop high-efficiency cultivation and production management in the tropics. 


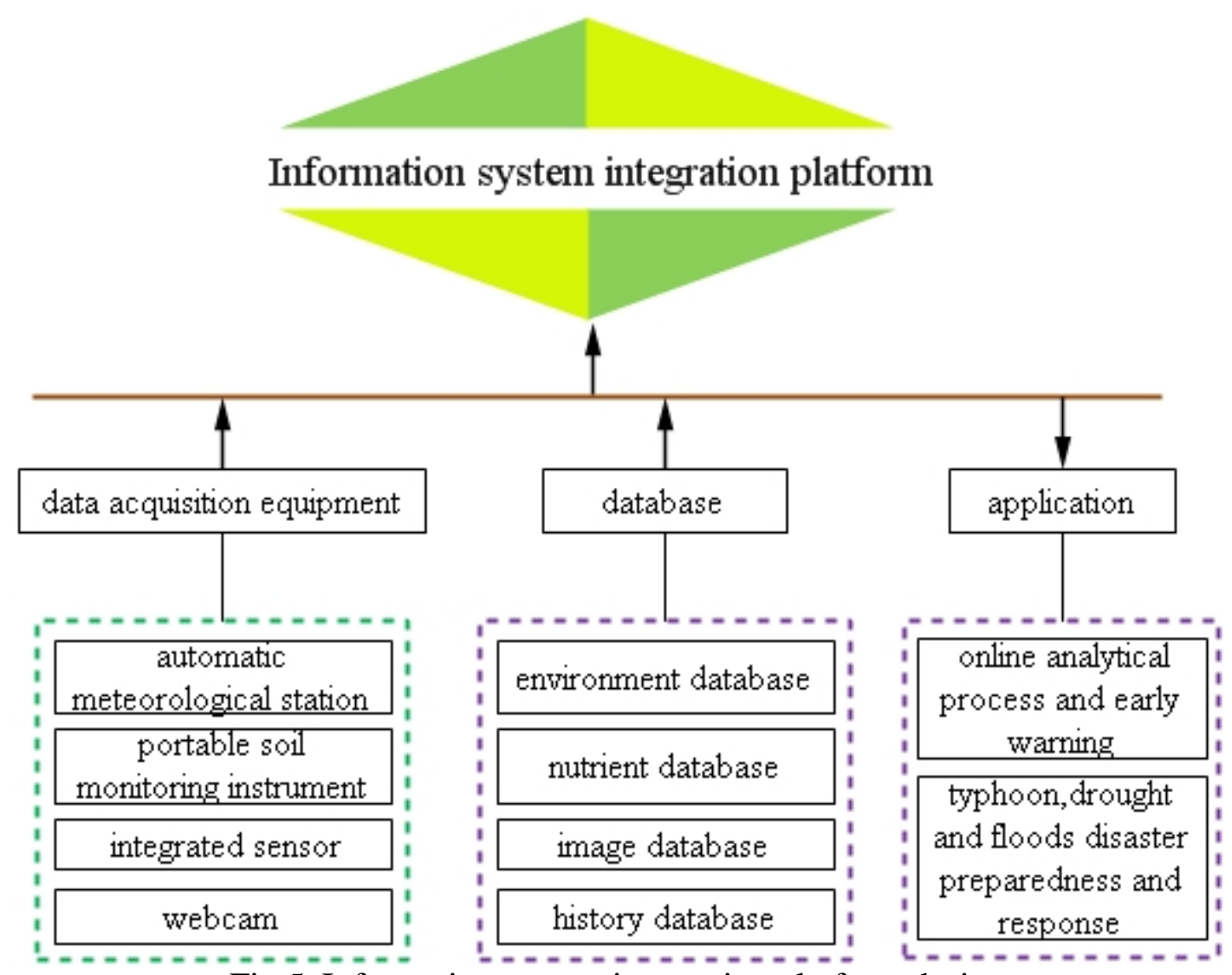

Fig.5. Information system integration platform design

\section{Acknowledgement}

In this paper, the research was sponsored by the Project of Technology Extension and Training of Tropical Crops of Ministry of Agriculture in 2016 and the key science and technology plan projects of Hainan Province(ZDXM2014082).

\section{References}

[1] Lingling Wang, Kun Lu, Hongxia Luo, Jihua Fang. The Key Technology Research on Information Acquisition about Tropical Crop Production Environment. Advanced Materials Research Vols. 989-994 (2014) pp 3452-3455.

[2] Lingling Wang, Hongxia Luo, Jianhua Cao, et al.The design of Intelligent Monitoring System on Tropical Greenhouse Crop Production Environment. Advanced Materials Research Vols. 1073-1076 (2015) pp 530-534.

[3] Jianhua Cao, Lingling Wang, Hongxia Luo, et al. A Research on Information Management System About the Field Environment of Tropical Crop, Advanced Materials Research Vols. 1073-1076 (2015) pp 3013-3016.

[4] Li Nan, Liu Chengliang, Li Yanming, et al. Development of remote monitoring system for soil moisture based on 3S technology alliance[J]. Transactions of the CSAE, 2010, 26(4): 169-174. (in Chinese with English abstract)

[5] Haiqing Yang. Modelling and Instrumentation of Soil and Plant Selected Properties Based on Visible-near-infrared Spectroscopy[D]. Zhejiang University(2011). (in Chinese with English abstract)

[6] Fang Hui. The research on field information on collection system based Palm-sized PC[D]. Zhejiang University(2013). (in Chinese with English abstract) 